\title{
Stimulation of the Frontal Eye Field Reveals Persistent Effective Connectivity after Controlled Behavior
}

\author{
Rei Akaishi, ${ }^{1}$ Yosuke Morishima, ${ }^{1}$ Vivian P. Rajeswaren, ${ }^{1}$ Shigeki Aoki, ${ }^{2}$ and Katsuyuki Sakai ${ }^{1}$ \\ Departments of ${ }^{1}$ Cognitive Neuroscience and ${ }^{2}$ Radiology, Graduate School of Medicine, The University of Tokyo, Bunkyo-ku, Tokyo 113-0033, Japan
}

Our ability to choose nonhabitual controlled behavior instead of habitual automatic behavior is based on a flexible control mechanism subserved by neural activity representing the behavior-guiding rule. However, it has been shown that the behavior slows down more when switching from controlled to automatic behavior than vice versa. Here we show that persistent effective connectivity of the neural network after execution of controlled behavior is responsible for the behavioral slowing on a subsequent trial. We asked normal human subjects to perform a prosaccade or antisaccade task based on a cue and examined the effective connectivity of the neural network based on the pattern of neural impulse transmission induced by stimulation of the frontal eye field (FEF). Effective connectivity during the task preparation period was dependent on the task that subjects had performed on the previous trial, regardless of the upcoming task. The strength of this persistent effective connectivity was associated with saccade slowing especially on trials after controlled antisaccade. In contrast, the pattern of regional activation changed depending on the upcoming task regardless of the previous task and the decrease in activation was associated with errors in upcoming antisaccade task. These results suggest that the effective connectivity examined by FEF stimulation reflects a residual functional state of the network involved in performance of controlled antisaccade and its persistence may account for the behavioral slowing on the subsequent trial.

\section{Introduction}

Our flexible behavior depends on cognitive control mechanism. It has been shown that sustained activity of neurons in the prefrontal cortex represents the rule of the task to be performed (Desimone and Duncan, 1995; Miller and Cohen, 2001). These neurons are thought to send control signals to other brain regions and update the connectivity of neural network according to the current task demand. This mechanism is critical when one has to override prepotent, automatic behavior and select nonhabitual, controlled behavior. Here the prefrontal control mechanism selects a weaker, task-appropriate response in the face of competition from an otherwise stronger but task-inappropriate one. Larger reaction times (RTs) in controlled behavior are taken to reflect neural processes associated with establishment of rule representation of that behavior, modulation of the network connectivity based on the rule, and resolution of the competition between controlled and automatic behavior.

Paradoxically, however, the increase in RTs has been shown to be larger when switching from controlled to automatic behavior

Received Dec. 15, 2009; revised Feb. 1, 2010; accepted Feb. 12, 2010.

This study was supported by a Grant-in-Aid for Young Scientists (S) from the Japan Society for the Promotion of Science, and a Grant-in-Aid for the Global Center of Excellence Program from the Ministry of Education, Culture, Sports, Science and Technology of Japan. We thank A. Mitsuto, M. Okamoto, R. Soga, K. Toma, and N. Yamamoto for help in conducting experiments, K. Morita for helpful discussion, and K. Duncan for proofreading this manuscript. R.A. designed the study, conducted the experiments, and analyzed the data. Y.M. and V.R. contributed to the experiments. Y.M. contributed to data analysis. S.A. contributed to the acquisition of MRl images. R.A. and K.S. wrote this paper.

Correspondence should be addressed to Katsuyuki Sakai, Department of Cognitive Neuroscience, Graduate School of Medicine, The University of Tokyo, 7-3-1 Hongo, Bunkyo-ku, Tokyo 113-0033, Japan. E-mail: ksakai@ m.u-tokyo.ac.jp.

DOI:10.1523/JNEUROSCI.6198-09.2010

Copyright $\odot 2010$ the authors $\quad 0270-6474 / 10 / 304295-11 \$ 15.00 / 0$ than vice versa. In a Stroop paradigm in which subjects switch between automatic word reading and controlled color naming tasks, a greater switch cost is observed on a word-reading trial after color naming than on a color-naming trial after word reading (Allport et al., 1994). Also, when switching between prosaccade and controlled antisaccade, that is, when switching between rules to make a saccade to and away from a visual target, a greater switch cost is observed on a prosaccade trial that follows an antisaccade trial than on an antisaccade trial that follows a prosaccade trial (Cherkasova et al., 2002; Fecteau et al., 2004; Barton et al., 2006). Moreover, responses become faster when switching from prosaccade to antisaccade than when repeating antisaccade. These results cannot be accounted for by the extra neural processing to establish the rule representation for controlled behavior, as this would result in an increase in RTs when switching from automatic to controlled behavior rather than vice versa. It is also shown that the effect cannot be eliminated with ample task preparation time of several seconds (Allport et al., 1994; Rogers and Monsell, 1995; Meiran, 2000; Barton et al., 2006), suggesting that a mechanism involved in the aftereffect of controlled behavior cannot be updated during a task preparation period on the next trial.

In the present study, we tested a possibility that the rule representation in the prefrontal cortex has been updated during task preparation but cannot fully reconfigure the pattern of network connectivity. Our hypothesis was that the network connectivity that has been modulated by the performance of controlled behavior persists during task preparation on the subsequent trial and influences behavior. To test the hypothesis, we asked normal human subjects to perform a prosaccade or antisaccade task and used a concurrent transcranial magnetic stimulation (TMS) and 
EEG technique to examine the pattern of effective connectivity, that is, efficiency of neural impulse transmission from one region to anatomically connected regions (Massimini et al., 2005; Driver et al., 2009; Morishima et al., 2009).

\section{Materials and Methods}

Subjects. For the main experiment of TMS over the frontal eye field (FEF), we recruited 22 healthy human subjects through poster and Internet. One subject was excluded from the analysis because his eye movements were too small to identify the saccade onset. The data from the remaining 21 subjects were used for analysis (11 males, 10 females; mean age 23.7; range 19-41). Additionally, we recruited 14 subjects for the of control site TMS experiment (3 females, 11 males; mean age: 26.7, range: $20-40)$. All subjects gave informed consent after the experimental procedures were explained. The study was approved by the ethics committee of the Graduate School of Medicine, the University of Tokyo.

Behavioral paradigm. Normal human subjects performed a prosaccade or antisaccade task based on a pretarget cue (Fig. $1 A$ ). Subjects fixated at the fixation point for a period of 1150-1450 ms. Subsequently the fixation point was replaced with a red or green cue, which instructed the subjects to perform an antisaccade or prosaccade, respectively (red and cyan in the figure for display purposes). The cue stayed on the screen for $100 \mathrm{~ms}$. At $500 \mathrm{~ms}$ after the cue onset, a target appeared for $100 \mathrm{~ms}$ at either the left or right of the center of the screen and the subjects made a saccade to or away from the target according to the cue. The subjects then kept fixating at the saccade endpoint until the reappearance of a fixation point $1400 \mathrm{~ms}$ later. Thus the interval between the offset of a target and presentation of a cue on a subsequent trial was $2550-2850 \mathrm{~ms}$. The white fixation, color cue, and white target were filled circles with a radius of $0.7^{\circ}$ of visual angle and the fixation and cue were presented at the center of the screen. Different trial types were presented in a pseudorandomized order so that an equal number of antisaccade and prosaccade trials, an equal number of TMS and no-TMS trials, an equal number of target positions, and an equal number of task switch and task repetition trials were present within each experimental session. The same saccade task could occur sequentially a maximum of three times. Subjects performed 10 or 12 experimental sessions, each containing 72 trials with a cue-target interval of $500 \mathrm{~ms}$ and six trials with cue-target interval of $250 \mathrm{~ms}$. Trials with a cue-target interval of $250 \mathrm{~ms}$ were included so that the subjects prepare for the upcoming saccade task immediately after presentation of a cue.

Eye movement. To encourage subjects to process the visual stimuli and compute saccade vectors on each trial, targets were presented at a position with eccentricity of 8.5, 10.2, or 11.9 degrees from the fixation point. Subjects were asked to make saccade toward the exact position of the target (prosaccade) or toward a position away from the target with the same eccentricity (antisaccade). Indeed, we found that the saccade amplitude changed depending on the variable eccentricity of the target for both prosaccade and antisaccade (supplemental Fig. 1, available at www. jneurosci.org as supplemental material). Before the EEG recording, subjects practiced the behavioral paradigm for $\sim 78$ trials. During these training sessions, eye movements were monitored on-line at a sampling
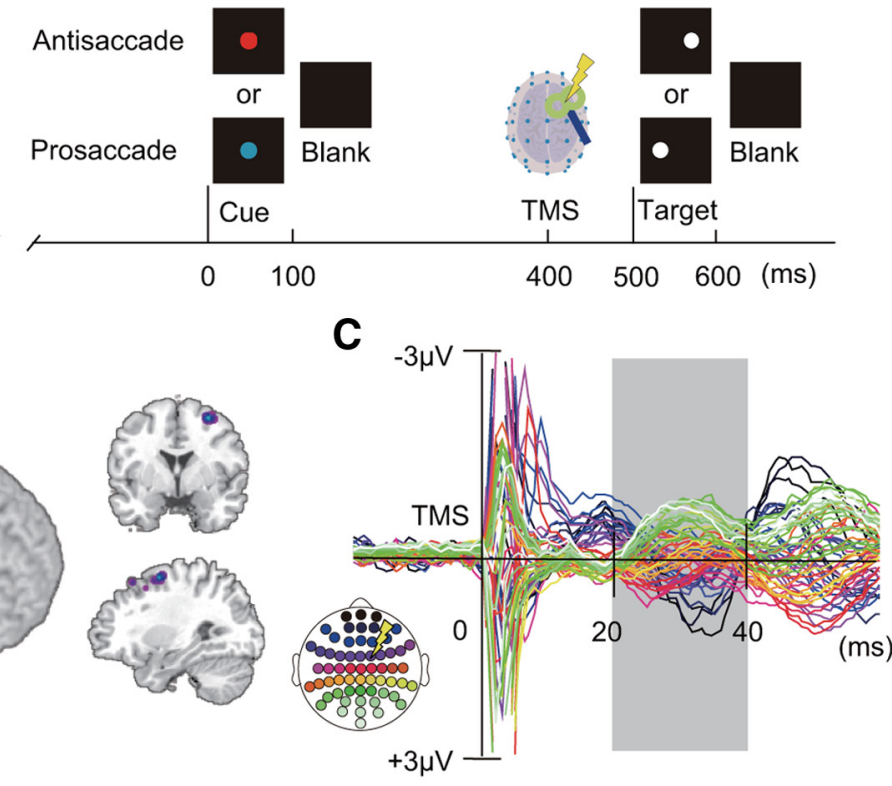

E

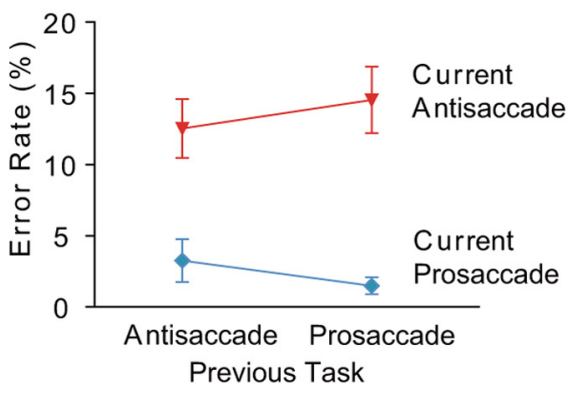

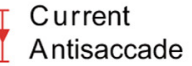

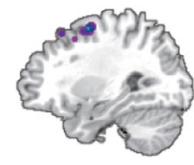

ms)

Figure 1. Cued saccade task and TMS-EPs. $\boldsymbol{A}$, Time line of the task. Subjects made prosaccade or antisaccade according to a pretarget cue (red cue: antisaccade, cyan cue: prosaccade). $\boldsymbol{B}$, Position of the FEF-TMS rendered on 3D surface of the MNI template conditions and all subjects, displayed from -20 to 60 ms of TMS. TMS-EPs were calculated by subtracting the EEG waveforms on MS trials from those on TMS trials. The analysis was focused on the time window of $20-40 \mathrm{~ms}$ after TMS (gray shading). Colors inset indicates scalp position at which TMS was applied. $\boldsymbol{D}, \boldsymbol{E}$, Latency of saccade onset (RTs) (D) and error rates (E), separately shown for the four conditions based on the task on the previous and current trials. Mean and SE across subjects are shown.

rate of $238 \mathrm{~Hz}$ using an iView eye tracker (SensoMotoric Instruments) and feedback was provided to ensure that they fixated on the center of the screen during the preparatory period. During the EEG recording sessions, horizontal EOGs were recorded with electrodes placed next to the lateral canthi of both eyes at a rate of $1450 \mathrm{~Hz}$. Saccade was defined as the rapid eye movement causing a sharp deflection of horizontal EOGs that exceeded $\pm 100 \mu \mathrm{V}$ from baseline within a time window of $500 \mathrm{~ms}$ after the onset of a visual target. Onsets of saccades were identified as the time point at which the second derivative of EOGs exceeded 4 SDs of the baseline. We also confirmed eye movements by recording the pupillary position of the left eye at a sampling rate of $60 \mathrm{~Hz}$ using ViewPoint eye tracker (Arrington Research).

TMS procedure. On half of the trials with a cue-target interval of 500 $\mathrm{ms}$, we gave a single-pulse low-intensity TMS at $400 \mathrm{~ms}$ after the onset of a task cue. TMS was not given on trials with a cue-target interval of 250 ms. TMS was applied with a figure-of-eight coil with a diameter of $70 \mathrm{~mm}$ and Magstim 200 stimulator (Magstim). We chose the FEF as the stimulation site for TMS because this region is shown to be involved in control of a voluntary saccade such as antisaccade (Munoz and Everling, 2004). The TMS coil was placed tangentially to the scalp over FC2 electrode with the handle of the coil pointing backwards and laterally $\sim 45$ degrees to the interhemispheric line. After the TMS-EEG session, structural MRIs were obtained from 12 subjects for the FEF-TMS sessions and 
7 subjects for the control site TMS sessions. The position of the TMS coil was then coregistered with the normalized structural brain images using a frameless stereotaxy system (Brainsight, Magstim). The TMS sites were located just anterior to the junction between the precentral sulcus and superior frontal sulcus on the right hemisphere (Fig. $1 B$ ). They were clustered at the coordinate $(30,2,57)$ in the standard brain of the Montreal Neurological Institute (MNI), which corresponds to the FEF (Ford et al., 2005; Grosbras et al., 2005; Brown et al., 2007). In the control experiment, the procedure was as above with the exception that we delivered TMS to the postcentral gyrus (mean coordinate: 28, -42, 66), following the procedure of a previous TMS-EEG study (Taylor et al., 2007b). The TMS intensity was fixed at 35\% of the maximum stimulator output. After the TMS-EEG session we measured the active motor threshold for each subject, which was defined as the lowest TMS intensity that evoked more than five small twitches of the left index finger in a series of 10 stimulations over the optimal position in the right primary motor cortex. For all the subjects, the TMS intensity used in the TMSEEG session did not exceed the active motor threshold: the TMS intensity ranged from $58 \%$ to $100 \%$ of active motor threshold (mean: $72 \%$ ). The TMS at this intensity did not induce muscle twitches in any body part when applied over the FEF. Here we used TMS as a probe to examine the functional state of the neural network without causing disruptive effect on behavior. TMS of similar intensities has been shown to induce neural impulse transmission from a stimulated region to distant cortical regions (Massimini et al., 2005; Morishima et al., 2009).

EEG recording. We recorded EEG with 60 electrodes placed according to an extended 10/20 system using TMS-compatible amplifiers (eXimia EEG, Nexstim; or BrainAmp, Brain Products). EEG signals were referenced to additional electrodes on the earlobes. For Nexstim the signals were bandpass filtered at $0.1-500 \mathrm{~Hz}$ and sampled at $1450 \mathrm{~Hz}$ with 16 bit resolution. For BrainAmp, the signals were low-pass filtered at $1000 \mathrm{~Hz}$, DC-corrected, and sampled at $2500 \mathrm{~Hz}$ with 16 bit resolution. Impedance of each electrode was kept below $5 \mathrm{k} \Omega$ for all experiments.

EEG data analysis. EEG data were preprocessed with Scan 4.3 Edit (Neuroscan) or BrainVision Analyzer (BrainProducts). We then used the EEGLAB toolbox (Delorme and Makeig, 2004) on Matlab (Mathworks) for re-referencing of the EEG to the mean of all electrodes, statistical analysis and data visualization. Trials with noise associated with blinking and EMG artifacts were automatically rejected using a threshold of average peak-to-peak amplitude of $70 \mu \mathrm{V}$ across channels during the interval of 200-400 ms after the cue-onset. The same rejection criterion was used for analysis of TMS-evoked potentials (TMS-EPs) and analysis of eventrelated potentials (ERPs). Trials with fixation break during $300 \mathrm{~ms}$ before target onset were present on 4.7\% (SD 9.0\%) of trials and were excluded from further analysis. For TMS-EP analysis, the waveforms were aligned to the onset of TMS or to the corresponding time point for no-TMS trials. For ERP analysis, the waveforms were aligned to the onset of the cue. The baseline correction was applied based on the pre-TMS interval of $60 \mathrm{~ms}$ for TMS-EP analysis, and on the precue interval of $200 \mathrm{~ms}$ for ERP analysis. The data analysis was conducted only on trials with a cue-target interval of $500 \mathrm{~ms}$.

TMS-evoked potentials. We took advantage of a concurrent TMS and EEG technique to examine the pattern of effective connectivity (Massimini et al., 2005; Driver et al., 2009; Morishima et al., 2009). The rationale of this technique is that stimulation applied over a particular brain region induces a current spread along anatomical connections in a manner dependent on the functional state of the interregional network connected with the stimulated region (Taylor et al., 2007b; Silvanto et al., 2008; Driver et al., 2009). The TMS induced significant changes in the scalp patterns of EEG potentials. We calculated TMS-EPs by subtracting the EEG waveforms on no-TMS trials from those on TMS trials (Fig. 1C; supplemental Fig. 2, available at www.jneurosci.org as supplemental material). We analyzed the data during the time window of $20-40 \mathrm{~ms}$ after TMS. This is to avoid artifacts caused by direct stimulation over EEG electrodes during $0-10 \mathrm{~ms}$ after TMS and also to avoid contamination of potentials evoked by auditory and tactile stimuli accompanying TMS, which occurs at $\sim 80 \mathrm{~ms}$ after TMS. Previous studies of cortical stimulation have shown that transmission of neural impulses from one cortical region to another at a neuronal population level takes $\sim 20-40 \mathrm{~ms}$
(Ilmoniemi et al., 1997; Massimini et al., 2005; Matsumoto et al., 2007; Morishima et al., 2009). In the present study we examined, for each electrode, the effect of the task that the subjects had performed on the previous trial (previous task) and the effect of the task that the subjects were about to perform on the current trial (current task) on the average of TMS-EP amplitude across the time window of 20-40 ms after TMS. To attenuate the problem of multiple comparisons and also to take into account the spatial smoothness in the distribution of scalp potentials, a significant task effect was determined when $p$ values of $<0.03$ were obtained on at least two neighboring electrodes. To verify the scalp distribution of the TMS-EPs observed in single-electrode analysis, we also examined three-way interactions between previous task (prosaccade and antisaccade), current task (prosaccade and antisaccade), and electrode positions (60 channels) on the TMS-EPs and ERPs. This analysis is free from the problem of multiple comparisons across electrodes.

Current source estimation. We used Curry 5.0 (Compumedics Neuroscan) for estimation of current source density that accounts for TMS-EPs and ERPs. We first estimated noise in our data by calculating the variance of the data in the pre-TMS interval of $60 \mathrm{~ms}(0.087 \mu \mathrm{V})$ or precue interval of $200 \mathrm{~ms}(0.084 \mu \mathrm{V})$. Based on the results of noise estimation, the sensor weighting and the regularization parameter of the current density reconstruction were determined. We then created the cortex model excluding the cerebellum with $6 \mathrm{~mm}$ resolution and 26,218 rotating dipoles and Boundary Element Model with three shells of fixed conductivities (scalp: $0.33 \mathrm{~S} / \mathrm{m}$; skull: $0.0042 \mathrm{~S} / \mathrm{m}$; brain: $0.33 \mathrm{~S} / \mathrm{m}$ ). The electrodes were rendered onto the scalp based on the coordinates acquired by the Brainsight frameless stereotaxy system. We estimated the current source density restricted to the cortex by using the minimum norm least squares (L2 Norm) method with correction of depth bias (Silva et al., 2004).

\section{Results}

\section{Behavioral data}

We first analyzed saccade latencies on correct response trials. Errors were defined as the saccades made in the direction opposite to that of the correct saccade based on electro-oculograms (EOGs). The data from 21 subjects were analyzed. There was a significant main effect of current task on saccade latencies (twoway repeated-measures ANOVA with factors of current task and previous task: $\left.F_{(1,20)}=110.7, p<0.001\right)$ : RTs were significantly longer on antisaccade trials than on prosaccade trials (Fig. 1D). We also found a significant main effect of the task on the previous trial $\left(F_{(1,20)}=21.64, p<0.001\right)$ : RTs were significantly longer on trials preceded by antisaccade than on trials preceded by prosaccade. Furthermore, we found asymmetry in the switch cost: RTs were significantly shorter when switching from prosaccade to antisaccade than when repeating antisaccade (paired $t$ test: $t_{(20)}=2.75$, $p=0.012$ ), whereas RTs were significantly longer when switching from antisaccade to prosaccade than when repeating prosaccade $\left(t_{(20)}=-3.15, p=0.005\right)$. Because of the task switch benefit on antisaccade and task switch cost on prosaccade, RTs did not differ significantly between switch and repetition trials when prosaccade and antisaccade trials were combined $\left(t_{(20)}=-1.33, p=0.20\right)$.

Error rates were significantly higher on antisaccade trials than on prosaccade trials (main effect of current task, $F_{(1,20)}=30.97$, $p<0.001$ ) (Fig. 1E). However, unlike RTs, error rates did not change significantly depending on the previous task type (main effect of previous task, $F_{(1,20)}=8.19, p=0.24$ ). We also found that the switch cost on error rates was symmetric: the increase in error rates when switching from antisaccade to prosaccade relative to repetition of prosaccade did not differ significantly from the increase in error rates when switching from prosaccade to antisaccade relative to repetition of antisaccade $\left(t_{(20)}=-1.21\right.$, $p=0.24$ ). Error rates were significantly higher on switch trials than on repetition trials when prosaccade and antisaccade trials were combined (paired $t$ test: $t_{(20)}=-2.83, p=0.010$ ). 
Importantly, the low-intensity TMS used in the present study did not affect the performance of the subjects. On RTs, there was no significant main effect of TMS $\left(F_{(1,20)}=3.60, p=0.072\right)$, no significant interaction between TMS and current task $\left(F_{(1,20)}=\right.$ $0.24, p=0.63)$, and no significant interaction between TMS and previous task $\left(F_{(1,20)}=0.81, p=0.38\right)$. Also the TMS did not induce eye movements, as confirmed by EOGs. For all of the previous studies on human subjects, TMS on the FEF does not induce eye movement even with maximum stimulator output (Müri et al., 1991; Wessel and Kömpf, 1991). In the present study we used far lower stimulation intensity.

\section{FEF effective connectivity reflects previous task}

Neural impulses induced by stimulation of the right FEF spread to distant cortical regions. After initial TMS-induced artifacts during $0-10 \mathrm{~ms}$ after a TMS-pulse, a negative change in the scalp potentials was observed on anterior electrodes at 10-20 ms (shown in black and blue in Fig. 1C). Then, during 20-40 ms after TMS, the scalp potentials showed negative deflection over posterior regions (shown in green and white in the figure) and positive deflection over frontal and central regions (shown in blue and pink in the figure). The temporal pattern of the changes in the current source density suggests that the TMS-induced current spread first to lateral parietal regions ipsilateral to the TMS site and then to the medial parietal and occipital regions (supplemental Fig. $3 A$, available at www.jneurosci.org as supplemental material). The current also spread to the contralateral prefrontal regions which may include the left FEF. These changes are likely to reflect TMS-induced neural impulse transmission from the right FEF to areas monosynaptically and multisynaptically connected with the stimulated region. Previous studies have also shown that TMS on the FEF induced changes in activation in superior parietal and medial parieto-occipital regions (Paus et al., 1997), anterior calcarine sulci and occipital poles (Ruff et al., 2006), and lateral parieto-occipital regions (Taylor et al., 2007a). In those studies it was difficult to specify the timing of TMS-induced modulation of activation because of the low temporal resolution of PET and fMRI (Paus et al., 1997; Ruff et al., 2006) or because of multiple TMS pulses delivered with an interval of $100 \mathrm{~ms}$ (Taylor et al., 2007a). In the present study we focused on the relatively short-latency response of the TMS-induced potentials which occurred at 20-40 ms after a single-pulse TMS. The observed effect is associated with direct neural impulse transmission induced by TMS (Silvanto et al., 2006; Morishima et al., 2009).

We first analyzed the data for correct trials. Waveforms of TMS-EPs were averaged within each subject for each condition of a two-by-two factorial design with factors of previous task type and current task type. This analysis was conducted on 19 subjects who made correct saccade responses on at least 40 trials for each condition. For each electrode, the average of TMS-EP amplitude across the time window of 20-40 ms after the TMS was entered into a two-way repeated-measures ANOVA across subjects. We found a significant main effect of the previous task on TMS-EPs (Fig. 2A,C). TMS-EPs on trials after antisaccade showed a positive deflection of potentials on the left anterior electrodes and a negative deflection on the bilateral posterior electrodes more dominantly on the right. In contrast, TMS-EPs on trials after prosaccade showed a negative deflection of potentials on the anterior medial electrodes in addition to a less prominent negative deflection on the right posterior electrodes compared with trials after antisaccade. Compared with trials after prosaccade, TMSEPs on trials after correct antisaccade were significantly more negative at posterior electrodes [two-way ANOVA: left posterior occipital electrode $(\mathrm{O} 1), F_{(1,18)}=7.66, p=0.013$; right posterior parietal electrode $(\mathrm{P} 4), F_{(1,18)}=7.70, p=0.013$ ].

We confirmed the significant effect of the previous task on the scalp distribution of the TMS-EPs by conducting a three-way repeated-measures ANOVA with factors of previous task (prosaccade and antisaccade), current task (prosaccade and antisaccade), and electrode positions (60 channels) on the TMS-EPs. We found a significant interaction between previous task types and electrode positions $\left(F_{(3.97,71.49)}=3.19, p=0.018\right.$, GreenhouseGeisser correction for violations of sphericity), which indicates a difference in the scalp distribution of TMS-EPs between trials after antisaccade and trials after prosaccade. This in turn suggests different cortical sources for trials after antisaccade and for trials after prosaccade. In fact, we found that the sources of the TMSEPs at 20-40 ms on trials after antisaccade were estimated to be in the ventral part of the lateral occipital regions on both hemispheres, whereas those on trials after prosaccade were in the medial parietal regions (supplemental Fig. 3B, available at www. jneurosci.org as supplemental material). Sources were also identified in bilateral ventral prefrontal regions for both the TMS-EPs on trials after prosaccade and antisaccade.

Importantly, the increase in the negative TMS-EPs on posterior electrodes on trials after correct antisaccade was observed regardless of the current task (main effect of current task: electrode O1, $F_{(1,18)}=0.34, p=0.57$; electrode $\mathrm{P} 4, F_{(1,18)}=$ $0.28, p=0.60)$. TMS-EPs did not differ significantly between switch and repetition trials either (interaction between previous and current tasks: electrode $\mathrm{O} 1, F_{(1,18)}=1.33, p=0.26$; electrode $\mathrm{P} 4$, $\left.F_{(1,18)}=0.33, p=0.57\right)$. Significant main effect of the current task and interaction between previous and current tasks were not observed at any electrode. We confirmed absence of significant effect of current task type on the spatial distribution of TMS-EPs by testing interactions between current task type and electrodes $\left(F_{(3.69,66.49)}=0.82, p=0.51\right.$, GreenhouseGeisser corrected). The three-way interaction between previous task, current task and electrodes was not significant, either $\left(F_{(3.65,65.73)}=0.42, p=0.78\right.$, Greenhouse-Geisser corrected $)$. Thus the residual effect of the previous task on TMS-EPs cannot be explained as reflecting control mechanism required to update rule representations in switching between tasks. Furthermore, neither the laterality of saccade direction nor saccade target on the previous trial influenced the TMS-EPs (supplemental Fig. 4, available at www.jneurosci.org as supplemental material) (saccade direction: electrode $\mathrm{O} 1, F_{(1,18)}=0.04, p=0.85$; electrode $\mathrm{P} 4, F_{(1,18)}=0.57, p=0.46$, saccade target: electrode $\mathrm{O} 1$, $F_{(1,18)}=0.15, p=0.71$; electrode $\left.\mathrm{P} 4, F_{(1,18)}=1.04, p=0.32\right)$.

Eye movements cannot account for the TMS-EPs because we excluded trials with eye movements during the cue-target interval. In addition, we confirmed that there was no significant main effect of previous task on EOGs during 20-40 ms after TMS (horizontal EOG electrodes, $F_{(1,18)}=0.34, p=0.57$, vertical EOG electrodes, $\left.F_{(1,18)}=2.29, p=0.15\right)$. Furthermore, the TMS did not induce head rotation, either, which would have produced asymmetric electromyographic responses of the neck muscles in occipital electrodes (Elsley et al., 2007). It is unlikely that repetition or alternation in the laterality of saccade target and saccade direction affected the TMS-EPs, because TMS-EPs were recorded before the presentation of a saccade target. Finally, the subjects were not able to anticipate the delivery of TMS pulse because stimulation was given in a pseudorandom manner. 

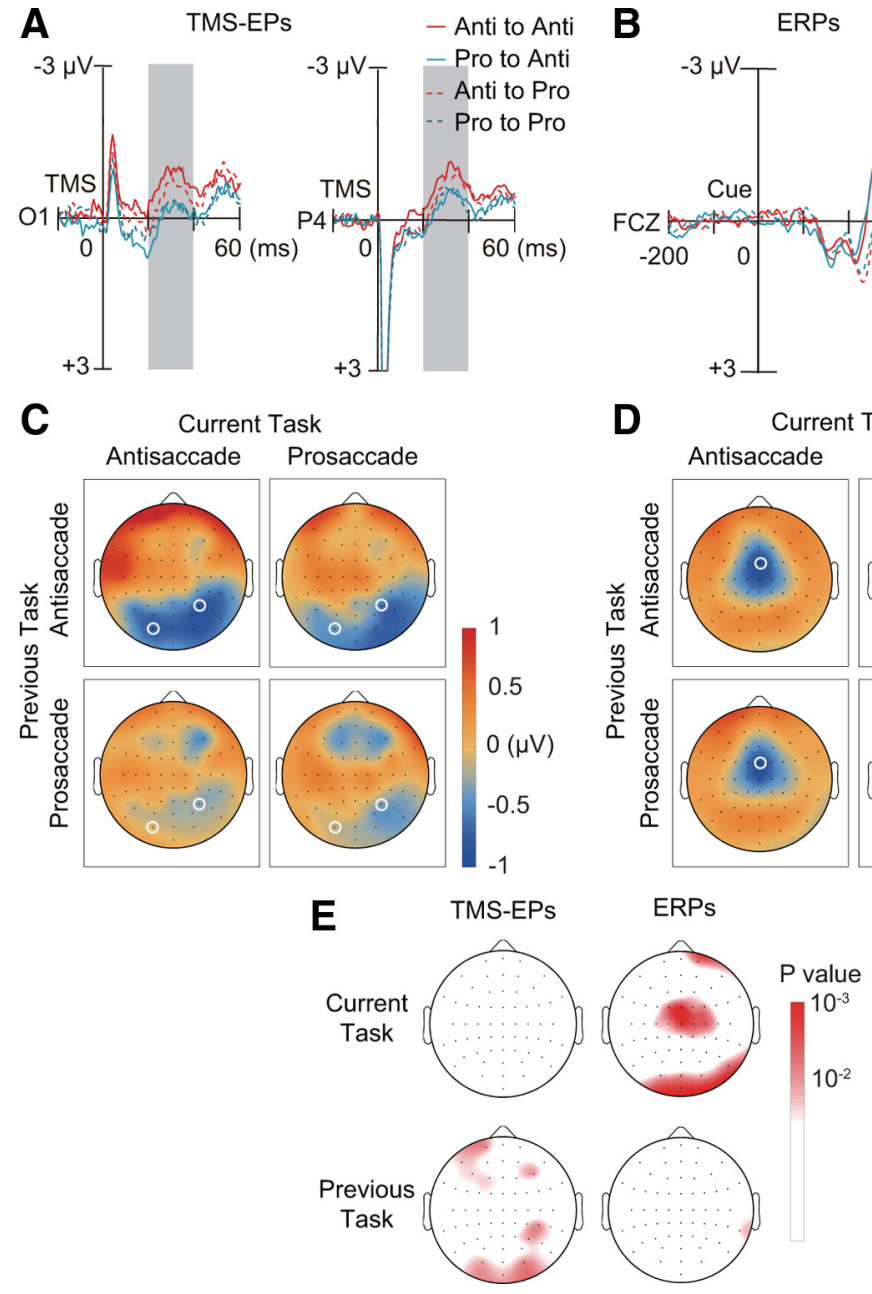

Figure 2. Dissociation between TMS-EPs and ERPs. $\boldsymbol{A}$, TMS-EPs at electrode 01 and P4 (marked with white circle in $\boldsymbol{C}$, separately shown for the four conditions based on the previous tasks (red: antisaccade; cyan: prosaccade) and current tasks (continuous line: antisaccade; dotted line: prosaccade). $\boldsymbol{B}$, ERPs on no-TMS trials at electrode FCZ (marked with white circle in $\boldsymbol{D}$ ). Convention same as in $\boldsymbol{A}$. C, Scalp topography of TMS-EPs amplitude within the time window of $20-40 \mathrm{~ms}$ after TMS (gray shading in $\boldsymbol{A}$ ), separately shown for previous task (rows) and current task (columns). Amplitudes were linearly interpolated across electrode positions and color-coded according to the color bar on the right. $D$, Scalp topography of ERPs amplitude within the time window of $420-440 \mathrm{~ms}$ after the cue onset (gray shading in $\boldsymbol{B}$ ), which corresponds to $20-40 \mathrm{~ms}$ after TMS on TMS trials. $\boldsymbol{E}$, Scalp topography of $p$ values based on two-way ANOVA with factors of current task (upper row) and previous task (lower row). Results are shown separately for TMS-EPs (left column) and ERPs (right column). The $p$ values are linearly interpolated across electrode positions and color-coded according to the color bar shown on the right.

\section{ERPs reflect upcoming task}

In contrast to TMS-EPs, ERPs as measured by conventional EEG analysis changed depending on the type of task that the subjects were preparing to perform. We examined ERPs on no-TMS trials during the time window of 420-440 ms after the cue onset, which corresponds to the time window of TMS-EPs analysis. We found that the waveforms at the anterior medial electrode (FCZ) were more negative on antisaccade trials than on prosaccade trials (Fig. $2 B, D$ ) (two-way ANOVA: $F_{(1,18)}=14.25, p=0.0014$ ). A significant difference in ERPs between antisaccade and prosaccade trials was observed on the central electrodes as early as 200$240 \mathrm{~ms}$ after the cue onset (supplemental Fig. 5, available at www.jneurosci.org as supplemental material). We next tested whether the current task type had a significant effect on the spatial pattern of ERPs. We found a significant interaction between current task types and electrode positions on ERPs during 420-440 ms after the cue onset ( $F=3.12, p=0.015$, Greenhouse-Geisser corrected). The cortical sources of the ERP during 400-440 ms after the task cue were estimated to be in the lateral and medial prefrontal cortex and inferior temporal and occipital regions in both tasks. On antisaccade trials compared with prosaccade trials, there was larger current source density in the dorsolateral prefrontal regions (supplemental Fig. 6, available at www.jneurosci. org as supplemental material).

Unlike TMS-EPs, ERPs were not affected by the previous task. ERPs on the anterior medial electrode were not modulated by the type of the previous task $\left(F_{(1,18)}=\right.$ $0.42, p=0.52$ ). The interaction between previous and current tasks was not significant, either $\left(F_{(1,18)}=2.03, p=0.17\right)$. Neither was the interaction between the previous task and electrode position nor the three-way interaction between previous task, current task, and electrode position significant $\left(F_{(2.52,45.40)}=0.84, p=0.46\right.$ and $F_{(4.14,74.55)}=0.93, p=0.45$, GreenhouseGeisser corrected, respectively). Thus, the difference in the baseline ERPs cannot account for the modulation of TMS-EPs by the previous task. The double dissociation between TMS-EPs and ERPs is clearly indicated by the scalp topographies of significance values in the two-way ANOVAs with factors of previous and current tasks (Fig. $2 E)$. The results suggest that during task preparation, the pattern of effective connectivity as assessed by TMS-EPs reflects previous task despite successful updating of task representation as measured by ERPs.

\section{Effective connectivity assessed by control site TMS does not show a task effect}

The effect of the previous task type was specific to the connectivity of the neural network involving the FEF. In the control experiment, we tested additional 14 subjects using the same experimental design but this time with stimulation delivered over the postcentral gyrus (mean coordinate: $28,-42,66$ ) (supplemental Fig. 7 , available at www. jneurosci.org as supplemental material). This site was chosen because it is not directly connected with the FEF. This site was also used as a control site for TMS in a previous study (Taylor et al., 2007a). The TMS-EPs after stimulation of the postcentral gyrus did not show a significant main effect of previous task at any electrode (electrode $\mathrm{O} 1, F_{(1,13)}=0.79, p=0.39$; electrode $\left.\mathrm{P} 4, F_{(1,13)}=0.13, p=0.73\right)$. Neither the effect of current task nor the interaction between previous and current task was significant (main effect of current task: electrode O1, $F_{(1,13)}=$ $3.68, p=0.077$; electrode $\mathrm{P} 4, F_{(1,13)}=1.93, p=0.19$, interaction: electrode $\mathrm{O} 1, F_{(1,13)}=0.03, p=0.87$; electrode $\mathrm{P} 4, F_{(1,13)}=0.009$, $p=0.93)$.

In this control experiment, the ERPs pattern on no-TMS trials changed significantly depending on the current task as in the FEF stimulation experiment: there was a significant main effect of current task on the anterior medial electrode $\mathrm{FCZ}\left(F_{(1,13)}=8.16\right.$, $p=0.013)$. The results suggest that the effect of previous task is 
A
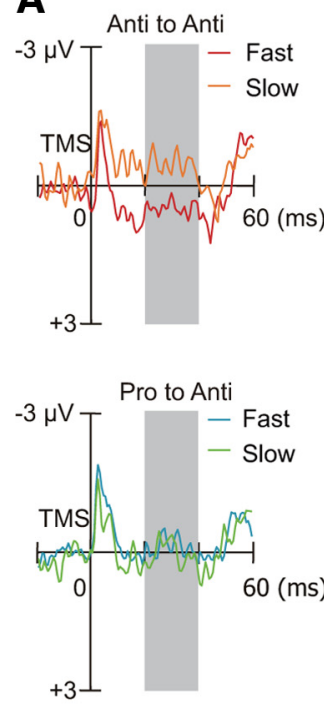

B
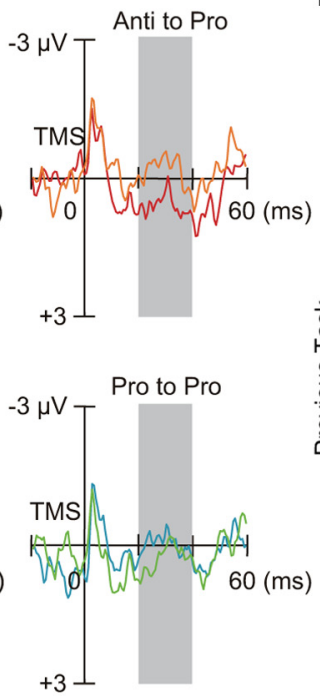

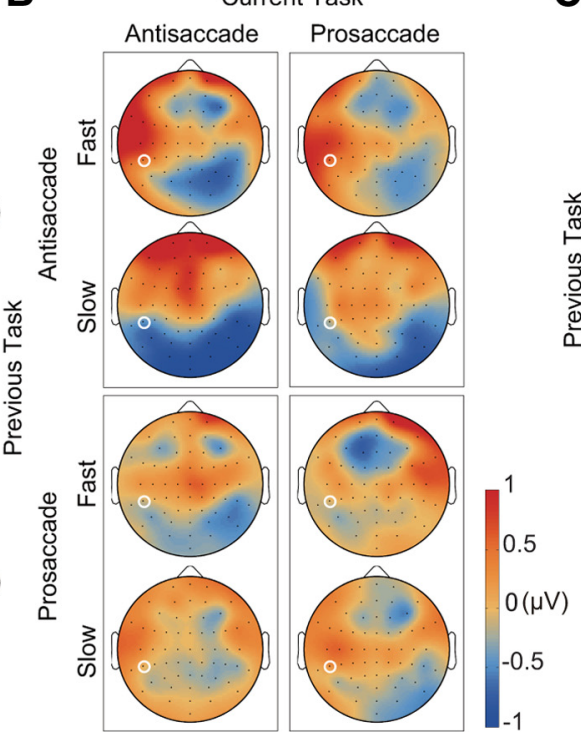

C Contrast: Fast vs. Slow

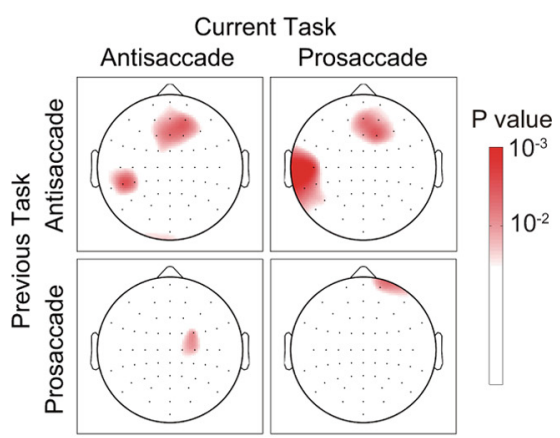

Figure 3. TMS-EPs are associated with saccade latency on trials after antisaccade. $\boldsymbol{A}$, TMS-EPs on fast and slow response trials at electrode TP7 (marked with white circle in $\boldsymbol{B}$ ), separately shown for the four conditions based on the previous and current tasks. $\boldsymbol{B}$, Scalp topography of TMS-EPs amplitude within the time window of $20-40 \mathrm{~ms}$ after TMS (gray shading in $\boldsymbol{A}$ ). In each cell defined by the previous task (rows) and current task (columns), maps are shown separately for fast (upper) and slow (lower) response trials. C, Scalp topography of $p$ values based on paired $t$ test. TMS-EPs at each electrode position were compared between fast and slow response trials. Results are shown separately for the types of previous task (rows) and types of current task (columns).

specific to the interregional network connected with the FEF. The significant effect of previous task on TMS-EPs was taken to reflect persistence of the connectivity pattern within the neural network involved in performance of the previous saccade task.

\section{Residual effective connectivity is associated with response slowing}

We reasoned that the residual network connectivity observed during task preparation period interfered with performance of the upcoming task. We further considered that the slowing of saccade response observed after antisaccade might be due to the residual of inhibitory control mechanism involved in the prior performance of antisaccade. To test the idea, for each condition in each subject, we divided trials into halves based on the median of the RTs in each condition and examined the relationship between TMS-EPs and RTs. The prediction was that larger TMSEPs would be associated with slower saccade responses after antisaccade. For this analysis we used the data from 15 subjects to ensure that at least 40 correct response trials were included for each of faster and slower response trials for each condition.

We first averaged TMS-EPs for each electrode, for each trial type, and for each subject and then subjected them to statistical analysis across subjects. As expected, we found that TMS-EPs were significantly more negative and spread more broadly to posterior electrodes on trials with slower saccade response than on trials with faster response (Fig. $3 A, B$ ). The topography for the slower response trials showed a positive deflection of potentials at the left anterior electrodes and a negative deflection at the bilateral posterior electrodes. In contrast, the topography for the faster response trials showed a negative deflection at the anterior medial electrodes in addition to a negative deflection at the right posterior electrodes. Thus the TMS-EPs on trials after antisaccade shown in the top row of Figure $2 C$ were the average of the two topography maps shown in the top panels of Figure $3 B$.

Crucially, the different patterns of topography for faster and slower response trials were only observed on trials after antisaccade. The difference in TMS-EPs between faster and slower trials was observed on left posterior temporal electrode (TP7) on trials after antisaccade regardless of current task (one-way ANOVA: antisaccade to antisaccade: $F_{(1,14)}=12.94, p=0.0029$, antisaccade to prosaccade: $\left.F_{(1,14)}=8.97, p=0.0096\right)$, but the difference was not significant on trials after prosaccade (prosaccade to antisaccade: $F_{(1,14)}=0.41, p=0.53$, prosaccade to prosaccade: $\left.F_{(1,14)}=0.95, p=0.35\right)$.

We confirmed the association between TMS-EPs and response time by conducting two sets of 3-way ANOVA with factors of response time (faster and slower responses), current task (prosaccade and antisaccade), and electrode position (60 channels), separately for trials after antisaccade and trials after prosaccade. We found a significant main effect of response time for trials after antisaccade $\left(F_{(1,14)}=6.90, p=0.020\right)$ but the effect was not significant for trials after prosaccade $\left(F_{(1,14)}=0.38, p=\right.$ $0.55)$. Interaction between electrode position and response time was not significant both for trials after antisaccade $\left(F_{(3.04,42.54)}=\right.$ $1.73, p=0.17$, Greenhouse-Geisser corrected) and for trials after prosaccade $\left(F_{(3.30,46.18)}=0.97, p=0.42\right.$, Greenhouse-Geisser corrected), suggesting similarity in the cortical distribution of current sources between fast and slow response trials after an antisaccade trial.

Scalp maps of significance value in one-way ANOVAs show a consistent pattern of differences in TMS-EPs between fast and slow response trials after antisaccade, regardless of current task (Fig. 3C, top row). In contrast, on trials after prosaccade, the difference in TMS-EPs between fast and slow response trials was less prominent: a significant difference was observed only at a single electrode position and this position differed depending on the current task (Fig. 3C, bottom row). In contrast to TMS-EPs, there was no significant difference in ERPs between fast and slow response trials for each condition of previous and current tasks (supplemental Fig. 8, available at www.jneurosci.org as supplemental material).

Analysis of the pattern of TMS-EPs across subjects also supports the idea that the residual network connectivity after performance of antisaccade affects the speed of the subsequent task 
A

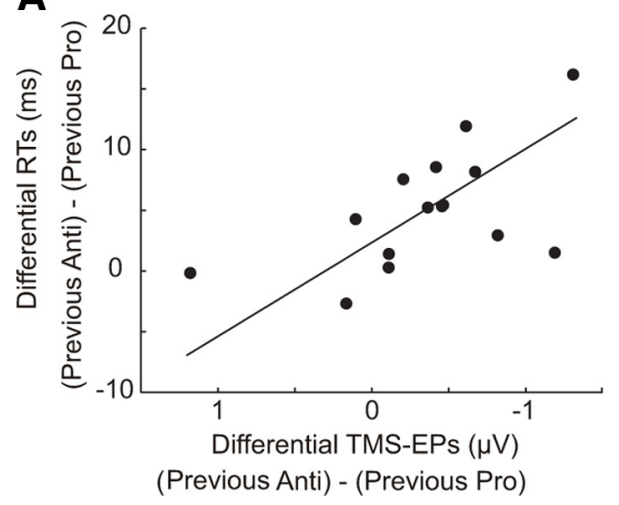

Figure 4. TMS-EPs are associated with across-subject variability in response slowing. $\boldsymbol{A}$, The difference in RTs between trials after antisaccade and trials after prosaccade is plotted against the difference in TMS-EPs between trials after antisaccade and trials after prosaccade at electrode TP8 (marked with white circle in $\boldsymbol{B}$ ) for 15 subjects. B, Scalp topography of correlation coefficient, $r$, between differential RTs and differential TMS-EPs across subjects. The correlation coefficient was calculated for each electrode position and linearly interpolated across electrode positions.

performance. We found that the differences in TMS-EPs between trials after antisaccade and prosaccade were significantly correlated with the differences in RTs between trials after antisaccade and prosaccade (Fig. 4). Subjects who showed more negative TMS-EPs on the right posterior electrodes on trials after antisaccade relative to those on trials after prosaccade responded more slowly on trials after antisaccade than on trials after prosaccade. A significant correlation between the differential TMS-EPs and RTs was found at the right temporal-parietal electrode TP8 (Pearson's $r=-0.58, p=0.024)$. Rank correlation was also significant, indicating that the relationship cannot be accounted for by the presence of outliers (Spearman's $\rho=-0.63, p=0.015$ ). At this electrode there was a significant difference in the TMS-EP between trials after antisaccade and trials after prosaccade (main effect of previous task: $F_{(1,18)}=5.21, p=0.035$ ) (Fig. $2 C$ ). The data suggest that the residual connectivity after performance of antisaccade interferes with subsequent saccade performance in terms of the response speed, which may explain the observed increase in saccade latency on trials after antisaccade compared with trials after prosaccade. However, the overlap between the spatial pattern of the across-subject correlation and that of the previous trial effect was only partial. For example, although the maximal correlation between the differential TMS-EPs and RTs was found at the right occipitotemporal electrode TP10 (Pearson's $r=$ $-0.63, p=0.012)$, the main effect of the previous task did not reach a significance level $\left(F_{(1,18)}=3.59, p=0.074\right)$. This might indicate that the residual of antisaccade includes not only the carryover of inhibitory control but also other components involved in antisaccade performance.

\section{Decrease in task-representing activation is associated with errors}

We next examined whether the residual effective connectivity can also account for errors in saccade response. We compared TMS-EPs between correct and error antisaccade trials from subjects who had at least 25 trials for both correct and error antisaccade $(n=6)$. We found that TMS-EPs on error antisaccade trials were not significantly different from those on correct antisaccade trials on the posterior electrode $\mathrm{P} 4$, where the effect of previous task was observed $\left(F_{(1,5)}=0.89, p=0.39\right)$ (Fig. 5A, $\left.C, E\right)$. For all the electrode positions, there was no significant difference in TMS-EPs between error antisaccade trials and correct antisaccade trials.
In contrast, there was significant difference in ERPs between trials of correct and error antisaccade. During the time window that corresponds to TMS-EPs (420-440 ms after the cue), the ERPs on the anterior medial electrode (FCZ) differed significantly between correct and error antisaccade trials $\left(F_{(1,5)}=11.0, p=0.021\right)$ (Fig. $5 B, D, E$ ). As shown in Figure 2, $B$ and $D$, there was a significant main effect of current task on the ERPs at this electrode. The results suggest that, in contrast to RTs, erroneous saccades could be due to a failure in establishing task-specific patterns of activation as measured by ERPs. Together with the behavioral data showing that error rates were not significantly affected by the previous task, the results may suggest that the residual connectivity plays a minor role in errors in controlled behavior, although the negative result could be due to lack of statistical power as a result of the small sample size.

\section{Persistent effective connectivity may result from neural competitions on the previous trial}

We so far have shown that the persistent connectivity of the neural network involving the FEF was significantly modulated by the previous task. A question remains, however, as to why the residual effect of the effective connectivity on saccade latency appeared only after antisaccade. We considered a possibility that the residual is due to the competition between reflexive and controlled saccade responses during performance of antisaccade. Antisaccade performance requires control mechanisms to bias the competition in favor of a weaker antisaccade response against a reflexive saccade response to the visual target (Schlag-Rey et al., 1997; Botvinick et al., 2001; Kristjánsson, 2007). In fact, the latency of erroneous prosaccade on antisaccade trials was significantly shorter than that of correct antisaccade (paired $t$ test: $\left.t_{(20)}=15.9, p<0.001\right)$ and was also significantly shorter than that of correct prosaccade $\left(t_{(20)}=5.58, p<0.001\right)$, suggesting that the errors were due to a failure to suppress a reflexive saccade response. Furthermore, on error trials in antisaccade condition, we observed a corrective saccade immediately following an erroneous reflexive saccade to the target on $90.0 \%$ (SD 12.0\%) of the error trials in antisaccade condition (Fig. 6A; supplemental Fig. 1 , available at www.jneurosci.org as supplemental material), which suggests parallel processing of reflexive saccade and controlled antisaccade (Schlag-Rey et al., 1997).

We then reasoned that, for those subjects who made a larger number of errors on antisaccade, there may be a higher degree of competitions between a reflexive saccade and controlled antisaccade even when they made correct antisaccade response. Such an increase in competitions and the demand to resolve the competitions may give rise to the modulation of effective connectivity. If so, it follows that the subjects who made larger number of errors on antisaccade trials show larger TMS-EPs on trials after correct antisaccade.

To investigate this possibility, we calculated the difference in TMS-EPs between trials after correct antisaccade and trials after correct prosaccade for each of the 19 subjects who made at least 40 correct antisaccade responses. The differential TMS-EPs can be taken to reflect the amount of residual network connectivity 
after antisaccade relative to prosaccade. Consistent with the prediction, we found a significant correlation between the differential TMS-EPs and the difference in error rates between antisaccade and prosaccade (Fig. $6 B, C$ ). Subjects who made a larger number of errors on antisaccade showed more negative TMS-EPs on trials after correct antisaccade at right posterior electrodes. The maximal correlation was found at the right centroparietal electrode, CP2 (Pearson's $r=0.56, p=0.013$ ). Rank correlation was also significant, indicating that the relationship did not depend on outliers (Spearman's $\rho=0.54, p=0.020$ ). However, at this electrode there was no significant difference in TMS-EP between trials after antisaccade and trials after prosaccade (main effect of previous task: $\left.F_{(1,18)}=0.88, p=0.36\right)$. At an adjacent electrode CP4, the across-subject correlation between differential TMS-EP and differential error rates was marginally significant (Pearson's $r=0.47, p=$ 0.041 , Spearman's $\rho=0.45, p=0.057$ ) and the main effect of previous task was also significant $\left(F_{(1,18)}=5.99, p=\right.$ $0.025)$. Thus, the scalp topography of the correlation coefficients overlapped only partially with the pattern of TMSEPs that reflects residual of the previous antisaccade (Fig. 2C), which may suggest that only a part of the persistent effective connectivity is associated with the competition.

Finally we examined TMS-EPs on trials after errors using the data from six subjects, from which we can obtain at least 25 trials for each of correct and error antisaccade. To increase the statistical power, we also included another three subjects who made errors on at least 25 antisaccade trials when trials with a cue-target interval of $250 \mathrm{~ms}$ were included. We found that TMS-EPs did not differ significantly between trials after correct and error antisaccade (posterior medial electrode POZ: $F_{(1,8)}=0.019, p=0.89$ ) (Fig. 7). TMS-EPs after error antisaccade showed a positive deflection of potentials on the left anterior electrodes and a negative deflection on the right posterior electrodes, in the same manner as in the TMS-EPs after correct antisaccade. In contrast, the pattern of TMS-EPs differed significantly between trials after correct prosaccade and trials after error antisaccade (electrode POZ: $F_{(1,8)}=7.26$, $p=0.027$ ).

The results suggest that TMS-EPs induced by FEF stimulation may reflect residual of the competitions between reflexive saccade and controlled antisaccade on a previous trial, regardless of whether the actual response was correct or an error. This idea is also supported by the behavioral data. RTs did not differ significantly between trials after correct antisaccade and trials after error antisaccade (main effect of error in antisaccade on the previous trial: $\left.F_{(1,20)}=0.29, p=0.60\right)$ and this was so regardless of the current task (interaction between current task and previous error: $\left.F_{(1,20)}=0.070, p=0.79\right)$.
B

ERPs

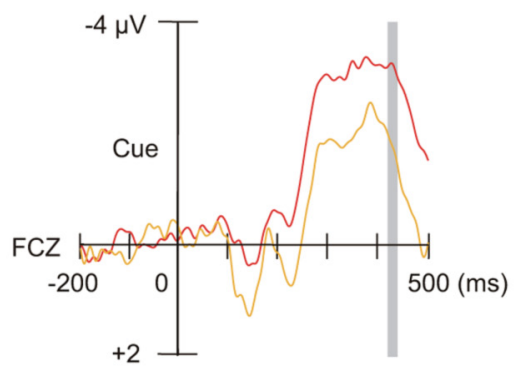

D

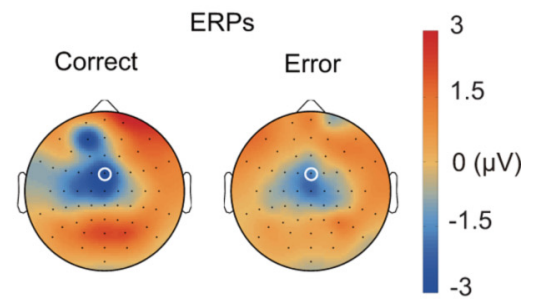

E
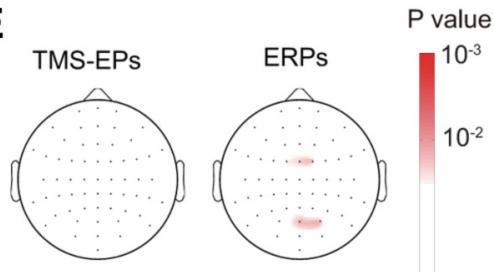

- Correct antisaccade

- Error antisaccade

$60(\mathrm{~ms})$

0.5

$0(\mu \mathrm{V})$

$-0.5$

$-1$

$-3$

Figure 5. TMS-EPs and ERPs on correct and error antisaccade trials. $\boldsymbol{A}$, TMS-EPs at electrode P4 (marked with white circle co the time window of $20-40 \mathrm{~ms}$ after of $p$ values based on comparison by one-way ANOVA between correct and error antisaccade trials. Results are shown separately for TMS-EPs (left) and ERPs (right).

\section{Discussion}

\section{Persistent effective connectivity and} task-representing activation

We found that stimulation of the FEF during the period of task preparation period induced different patterns of TMS-EPs depending on whether the subjects had performed a prosaccade or antisaccade on the previous trial. In contrast, scalp patterns of ERPs changed depending on the current task regardless of the previous task. Our cortical source density analysis on the TMSEPs suggests that the TMS-induced impulse was transmitted from the right FEF to anatomically connected regions such as parietal areas on the same hemisphere and then to more distant regions such as bilateral ventral occipital areas (Cavada and Goldman-Rakic, 1989; Stanton et al., 1995). Though both TMSEPs and ERPs are considered to be generated by summation of EPSPs, TMS-EPs reflect effective connectivity of the neural network involving the stimulated region, whereas ERPs reflect neural activation in a set of localized regions.

Effective connectivity has been shown to be modulated by synchronous activity of neurons between brain areas. A singleunit recording study on monkeys has shown that an attentionrelated increase of activity in FEF neurons is followed by an increase in the causal influence of the FEF over V4 with a delay of several tens of milliseconds (Gregoriou et al., 2009). Also in fMRI studies on human subjects, an increase in correlation of activa- 
A

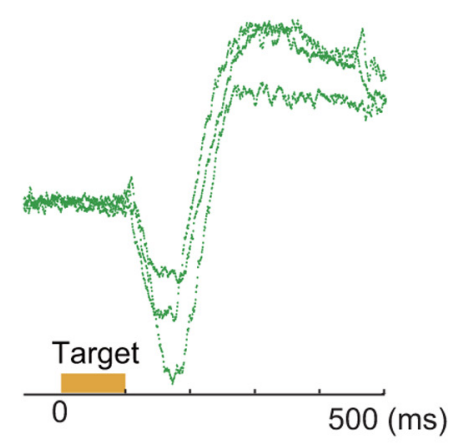

B

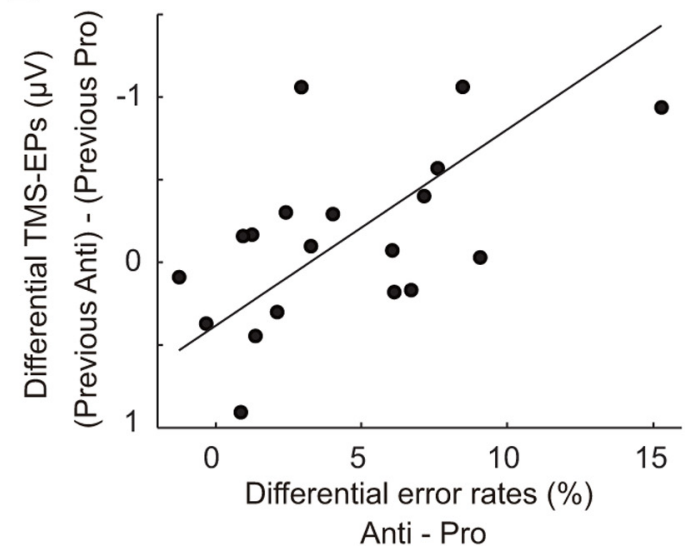

C

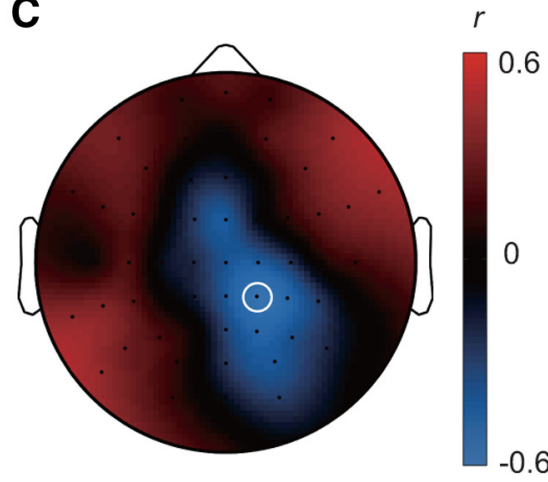

Figure 6. TMS-EPs after correct antisaccade are correlated with error rates across subjects. $\boldsymbol{A}$, Representative EOG traces for erroneous reflexive saccade immediately followed by corrective antisaccade. $\boldsymbol{B}$, The difference in TMS-EPs at electrode CP2 (marked with white circle in $\boldsymbol{C}$ ) between trials after antisaccade and trials after prosaccade is plotted against the difference in error rates between antisaccade and prosaccade trials for 19 subjects. $C$, Scalp topography of correlation coefficient, $r$, between differential TMS-EPs and error rates calculated for each electrode position across subjects.

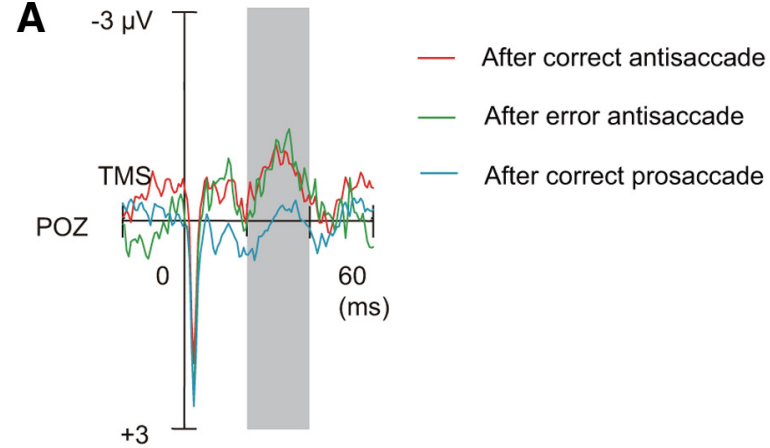

B

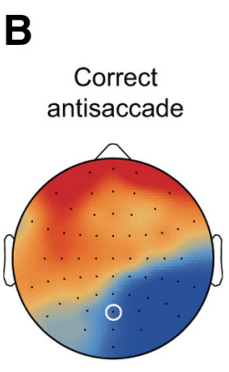

Previous trial
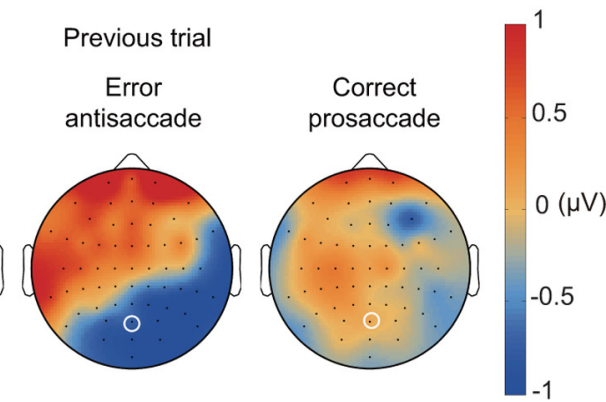

C

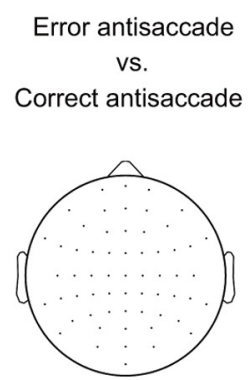

Error antisaccade

$P$ value vs.

Correct prosaccade

$10^{-2}$

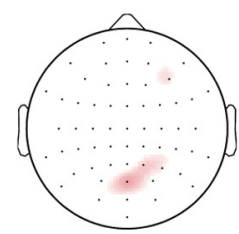

Figure 7. TMS-EPs on trials after correct antisaccade, error antisaccade, and correct prosaccade. $\boldsymbol{A}$, TMS-EPs at electrode POZ (marked with white circle in $\boldsymbol{B}$ ) on trials after correct antisaccade, error antisaccade, and correct prosaccade. $\boldsymbol{B}$, Scalp topography of TMS-EPs amplitude, separately shown for trials after correct antisaccade (left), error antisaccade (center), and correct prosaccade (right). C, Scalp topography of $p$ values based on comparison by one-way ANOVA between trials after error antisaccade and correct antisaccade (left) and between trials after error antisaccade and correct prosaccade (right). tion is observed between areas that show an increase of activation during task preparation (Sakai and Passingham, 2003, 2006). Together, these previous results suggest that the effective connectivity is modulated by the activity of neurons that reflects the task at hand.

In the present study, however, the effective connectivity of neural network involving the FEF was modulated by the previous task rather than by the task at hand. The results suggest that the FEF network connectivity is not modulated by on-going neural activity representing the task to be performed, but retains the memory of the previous task that had been performed several seconds before. This seems in contrast to the idea that cognitive control mechanisms are achieved by interplay between activity of rule-representing neurons and network connectivity. We suggest that the network connected with more anterior prefrontal areas in which neurons represent abstract rules may be updated according to the upcoming task (Miller and Cohen, 2001; Koechlin et al., 2003; Sakai and Passingham, 2003, 2006). In contrast, the network connected with the FEF may be more directly involved in execution of a controlled saccade and retains the memory of previous performance. Of note is that the persistent effective connectivity of the FEF network did not differ depending on either the direction of saccade or the laterality of the saccade target, suggesting that the FEF network does not retain the memory for specific motor command nor the memory for specific visual stimulus.

\section{Persistent effective connectivity is responsible for response slowing}

We have further shown that a larger amount of persistent effective connectivity was associated with slowing of saccade responses on trials after antisaccade. However, the association was not observed on trials after prosaccade. It has been argued that response slowing on trials after antisaccade is due to a carryover of a general inhibitory control mechanism over a saccade response system rather than due to requirement of inhibiting a previous task set (Cherkasova et al., 2002; Barton et al., 2006). The effective connectivity pattern that we observed in the present study may reflect such a carryover of an inhibitory control mechanism subserved by the neural network involving the FEF.

Although the spatial pattern of the TMS-EPs associated with response slowing on the subsequent trial was similar to the pat- 
tern of the TMS-EPs that reflects the previous task, the maximal effect of the behavioral slowing was observed at different electrode positions. This may indicate that only a part of the residual FEF network connectivity is associated with behavior. Concurrent use of TMS and fMRI may be useful to identify the behaviorally relevant neural structures that receive inputs from the FEF (Ruff et al., 2006).

We also found that, whereas the slowing of saccade response was associated with changes in TMS-EPs but not with ERPs, errors were associated with changes in ERPs. The pattern of ERPs reflecting the current antisaccade task was significantly reduced on error trials, which may reflect a decrease in preparatory activity of antisaccade neurons in the supplementary eye field (SchlagRey et al., 1997; Ford et al., 2005) or decrease in tonic pretarget activity of fixation neurons in the FEF (Lo et al., 2009).

\section{Reactive mechanisms of network modulation}

Our results suggest that full configuration of a task-specific pattern of network connectivity may take place only during actual performance of that task. Execution of antisaccade requires interactions between sensory signals induced by the target and control signals involved in suppression of a reflexive saccade to a visual target. Consistent with the idea, microstimulation of the FEF in monkeys has modulatory influence over the activity in visual areas when a visual target is presented (Moore and Armstrong, 2003; Ekstrom et al., 2008). Also the pattern of TMS-EPs after stimulation of the FEF has been shown to be modulated according to the current task demands when the TMS was given after the presentation of a visual target (Morishima et al., 2009). These observations imply that while activation in some brain areas can represent abstract rules during task preparation, modulation of the network connectivity directly involved in task execution occurs by reacting to the sensory bottom-up signals. The reactive nature of network modulation is consistent with the idea of stimulus-triggered control process (Rogers and Monsell, 1995). On the other hand, the persistence of effective connectivity pattern from the previous trial is consistent with the idea of a carryover of the previous task set (Allport et al., 1994; Wylie and Allport, 2000).

Our results also suggest that the reactive mechanism of network modulation may result from the control mechanism involved in resolution of competition between reflexive and controlled responses during performance of antisaccade (Botvinick et al., 2001; Kristjánsson, 2007). Subjects who make a large number of errors on antisaccade are thought of as having higher level of the competitions due to greater tendency to make a reflexive saccade toward a visual target. Indeed, we found larger TMS-EPs on trials after antisaccade on those subjects. Turnaround saccades observed on most of the error antisaccade trials indicate the presence of competitions regardless of whether the subjects made correct antisaccade or erroneous reflexive saccade and we found similar amounts of residual connectivity between trials after correct antisaccade and trials after erroneous reflexive saccades. The competition is thought to occur at a response stage of processing during task execution and we consider that the effective connectivity revealed by FEF stimulation reflects residual of the competitions at this stage. However, the results that support the neural competition account were based on across-subject correlation with lenient statistical criteria, and thus the confirmation of these findings awaits further investigation.

\section{Neurobiological mechanism of persistent effective connectivity}

There are several possible mechanisms for the task-dependent modulation of the TMS-EPs. One possibility is that, depending on the previous task, different populations of neurons within the stimulated region show sustained activity during the intertrial interval as well as the task preparation period on the subsequent trial (Mansouri et al., 2007; Histed et al., 2009). The modulation in the pattern of TMS-EPs may reflect the difference in regions to which these neurons project. Because of the low spatial resolution and baseline correction of ERPs, we may have failed to detect differential activation of neurons associated with prior performance of antisaccade and prosaccade in our ERP data. We, however, think this possibility less likely because we found a significant difference in ERPs between preparation of antisaccade and prosaccade and yet TMS-EPs at the same timing changed depending on the previous task rather than the task that the subjects were preparing to perform.

Another possibility that we favor is that the modulation of the TMS-EPs reflects changes in the efficacy of synaptic transmission within the network involving the stimulated region. The effective connectivity pattern that reflects the previous task can be considered as reflecting use-dependent modulation of synaptic efficacy. In the present study, the persistent effective connectivity was shown to be maintained for a three-second intertrial interval, which suggests that the underlying mechanism must be able to sustain the network state over this interval. This may be achieved by synaptic mechanism with slow temporal dynamics (Carter and Wang, 2007; Mongillo et al., 2008) or by neuromodulators such as noradrenalin and dopamine (Aston-Jones and Cohen, 2005; Esser et al., 2009).

The present results suggest that the interregional network directly involved in task execution retains the memory of the previous task performance through modulation of the connectivity weighting even when activation in some brain regions represents the current task demand. The results further suggest that the persistent network state might be a result of control mechanisms essential for goal-directed behavior. Future studies are required to investigate whether the present findings on saccade tasks can be generalized to other cognitive functions.

\section{References}

Allport A, Styles EA, Hsieh SL (1994) Shifting intentional set: exploring the dynamic control of tasks. In: Attention and performance, Vol. XV (Umilta C, Moscovitch M, eds), pp 421-452. Cambridge, MA: MIT.

Aston-Jones G, Cohen JD (2005) An integrative theory of locus coeruleusnorepinephrine function: adaptive gain and optimal performance. Annu Rev Neurosci 28:403-450.

Barton JJ, Greenzang C, Hefter R, Edelman J, Manoach DS (2006) Switching, plasticity, and prediction in a saccadic task-switch paradigm. Exp Brain Res 168:76-87.

Botvinick MM, Braver TS, Barch DM, Carter CS, Cohen JD (2001) Conflict monitoring and cognitive control. Psychol Rev 108:624-652.

Brown MR, Vilis T, Everling S (2007) Frontoparietal activation with preparation for antisaccades. J Neurophysiol 98:1751-1762.

Carter E, Wang XJ (2007) Cannabinoid-mediated disinhibition and working memory: dynamical interplay of multiple feedback mechanisms in a continuous attractor model of prefrontal cortex. Cereb Cortex 17:i16-i26.

Cavada C, Goldman-Rakic PS (1989) Posterior parietal cortex in rhesus monkey: II. Parcellation of areas based on distinctive limbic and sensory corticocortical connections. J Comp Neurol 287:422-445.

Cherkasova MV, Manoach DS, Intriligator JM, Barton JJ (2002) Antisaccades and task-switching: interactions in controlled processing. Exp Brain Res 144:528-537.

Delorme A, Makeig S (2004) EEGLAB: an open source toolbox for analysis of single-trial EEG dynamics including independent component analysis. J Neurosci Methods 134:9-21. 
Desimone R, Duncan J (1995) Neural mechanism of selective visual attention. Annu Rev Neurosci 18:193-222.

Driver J, Blankenburg F, Bestmann S, Vanduffel W, Ruff CC (2009) Concurrent brain-stimulation and neuroimaging for studies of cognition. Trends Cogn Sci 13:319-327.

Ekstrom LB, Roelfsema PR, Arsenault JT, Bonmassar G, Vanduffel W (2008) Bottom-up dependent gating of frontal signals in early visual cortex. Science 321:414-417.

Elsley JK, Nagy B, Cushing SL, Corneil BD (2007) Widespread presaccadic recruitment of neck muscles by stimulation of the primate frontal eye fields. J Neurophysiol 98:1333-1354.

Esser SK, Hill S, Tononi G (2009) Breakdown of effective connectivity during slow wave sleep: investigating the mechanism underlying a cortical gate using large-scale modeling. J Neurophysiol 102:2096-2111.

Fecteau JH, Au C, Armstrong IT, Munoz DP (2004) Sensory biases produce alternation advantage found in sequential saccadic eye movement tasks. Exp Brain Res 159:84-91.

Ford KA, Goltz HC, Brown MR, Everling S (2005) Neural processes associated with antisaccade task performance investigated with event-related FMRI. J Neurophysiol 94:429-440.

Gregoriou GG, Gotts SJ, Zhou H, Desimone R (2009) High-frequency, long-range coupling between prefrontal and visual cortex during attention. Science 324:1207-1210.

Grosbras MH, Laird AR, Paus T (2005) Cortical regions involved in eye movements, shifts of attention, and gaze perception. Hum Brain Mapp 25:140-154.

Histed MH, Pasupathy A, Miller EK (2009) Learning substrates in the primate prefrontal cortex and striatum: sustained activity related to successful actions. Neuron 63:244-253.

Ilmoniemi RJ, Virtanen J, Ruohonen J, Karhu J, Aronen HJ, Näätänen R, Katila T (1997) Neuronal responses to magnetic stimulation reveal cortical reactivity and connectivity. Neuroreport 8:3537-3540.

Koechlin E, Ody C, Kouneiher F (2003) The architecture of cognitive control in the human prefrontal cortex. Science 302:1181-1185.

Kristjánsson A (2007) Saccade landing point selection and the competition account of pro- and antisaccade generation: the involvement of visual attention-a review. Scand J Psychol 48:97-113.

Lo CC, Boucher L, Paré M, Schall JD, Wang XJ (2009) Proactive inhibitory control and attractor dynamics in countermanding action: a spiking neural circuit model. J Neurosci 29:9059-9071.

Mansouri FA, Buckley MJ, Tanaka K (2007) Mnemonic function of the dorsolateral prefrontal cortex in conflict-induced behavioral adjustment. Science 318:987-990.

Massimini M, Ferrarelli F, Huber R, Esser SK, Singh H, Tononi G (2005) Breakdown of cortical effective connectivity during sleep. Science 309:2228-2232.

Matsumoto R, Nair DR, LaPresto E, Bingaman W, Shibasaki H, Lüders HO (2007) Functional connectivity in human cortical motor system: a cortico-cortical evoked potential study. Brain 130:181-197.

Meiran N (2000) Modeling cognitive control in task-switching. Psychol Res 63:234-249.
Miller EK, Cohen JD (2001) An integrative theory of prefrontal cortex function. Annu Rev Neurosci 24:167-202.

Mongillo G, Barak O, Tsodyks M (2008) Synaptic theory of working memory. Science 319:1543-1546.

Moore T, Armstrong KM (2003) Selective gating of visual signals by microstimulation of frontal cortex. Nature 421:370-373.

Morishima Y, Akaishi R, Yamada Y, Okuda J, Toma K, Sakai K (2009) Taskspecific signal transmission from prefrontal cortex in visual selective attention. Nat Neurosci 12:85-91.

Munoz DP, Everling S (2004) Look away: the anti-saccade task and the voluntary control of eye movement. Nat Rev Neurosci 5:218-228.

Müri RM, Hess CW, Meienberg O (1991) Transcranial stimulation of the human frontal eye field by magnetic pulses. Exp Brain Res 86:219-223.

Paus T, Jech R, Thompson CJ, Comeau R, Peters T, Evans AC (1997) Transcranial magnetic stimulation during positron emission tomography: a new method for studying connectivity of the human cerebral cortex. J Neurosci 17:3178-3184.

Rogers RD, Monsell S (1995) Costs of a predictable switch between simple cognitive tasks. J Exp Psychol Gen 124:207-231.

Ruff CC, Blankenburg F, Bjoertomt O, Bestmann S, Freeman E, Haynes JD, Rees G, Josephs O, Deichmann R, Driver J (2006) Concurrent TMSfMRI and psychophysics reveal frontal influences on human retinotopic visual cortex. Curr Biol 16:1479-1488.

Sakai K, Passingham RE (2003) Prefrontal interactions reflect future task operations. Nat Neurosci 6:75-81.

Sakai K, Passingham RE (2006) Prefrontal set activity predicts rule-specific neural processing during subsequent cognitive performance. J Neurosci 26:1211-1218.

Schlag-Rey M, Amador N, Sanchez H, Schlag J (1997) Antisaccade performance predicted by neuronal activity in the supplementary eye field. Nature 390:398-401.

Silva C, Maltez JC, Trindade E, Arriaga A, Ducla-Soares E (2004) Evaluation of L1 and L2 minimum norm performances on EEG localizations. Clin Neurophysiol 115:1657-1668.

Silvanto J, Lavie N, Walsh V (2006) Stimulation of the human frontal eye fields modulates sensitivity of extrastriate visual cortex. J Neurophysiol 96:941-945.

Silvanto J, Muggleton N, Walsh V (2008) State-dependency in brain stimulation studies of perception and cognition. Trends Cogn Sci 12:447-454

Stanton GB, Bruce CJ, Goldberg ME (1995) Topography of projections to posterior cortical areas from the macaque frontal eye fields. J Comp Neurol 353:291-305.

Taylor PC, Nobre AC, Rushworth MF (2007a) FEF TMS affects visual cortical activity. Cereb Cortex 17:391-399.

Taylor PC, Nobre AC, Rushworth MF (2007b) Subsecond changes in topdown control exerted by human medial frontal cortex during conflict and action selection: a combined transcranial magnetic stimulation-electroencephalography study. J Neurosci 27:11343-11353.

Wessel K, Kömpf D (1991) Transcranial magnetic stimulation: lack of oculomotor response. Exp Brain Res 86:216-218.

Wylie G, Allport A (2000) Task switching and the measurement of "switch costs." Psychol Res 63:212-233. 